\title{
Butein Activates Autophagy Through AMPK/TSC2/ULK1/mTOR Pathway to Inhibit IL-6 Expression in IL-1 $\beta$ Stimulated Human Chondrocytes
}

\author{
Mohammad Y Ansaria Nashrah Ahmad ${ }^{\mathrm{b}}$ Tariq M Haqqia \\ aDepartment of Anatomy and Neurobiology, Northeast Ohio Medical University, Rootstown, bSchool of \\ Biomedical Sciences, Kent State University, Kent, USA
}

\section{Key Words}

Butein • Osteoarthritis • Autophagy • Inflammation • AMPK • mTOR • TSC2 • ULK1

\begin{abstract}
Background/Aims: Butein (2',3,4,4'-Tetrahydroxychalcone), a polyphenol produced by several plants including Butea monoserpma, has been reported to exert potent anti-inflammatory activity but the mechanism remains unknown. In the present work we investigated the mechanism of Butein-mediated suppression of IL-6 expression in normal and human osteoarthritis (OA) chondrocytes under pathological conditions. Methods: Expression level of interleukin-6 (IL-6) protein in OA cartilage was analyzed by immunohistochemistry using a validated antibody. Chondrocytes derived from normal or OA cartilage by enzymatic digestion were pretreated with Butein followed by stimulation with interleukin-1 $\beta$ (IL-1 $\beta$ ) and the levels of IL- 6 mRNA were quantified by TaqMan assay and the protein levels were measured by Western immunoblotting. Autophagy activation was determined by Western blotting and confocal microscopy. Autophagy was inhibited by siRNA mediated knockdown of ATG5. Results: Expression of IL-6 protein was high in the OA cartilage compared to smooth cartilage from the same patient. OA chondrocytes and cartilage explants stimulated with IL-1 $\beta$ showed high level expression of IL-6 mRNA and protein. Butein increased the phosphorylation of AMPKa ${ }^{\text {Thr-172, }}$ TSC2 $^{\text {Ser-1387 }}$ and ULK1 ${ }^{\text {Ser-317 }}$ and inhibited the phosphorylation of mTOR ${ }^{\text {Ser-2448 }}$ and its downstream target p70S6K and increased autophagy flux that correlated with the suppression of the IL-1 $\beta$ mediated expression of IL- 6 in normal and OA chondrocytes. In OA chondrocytes with siRNA-mediated knockdown of ATG5 expression, treatment with Butein failed to activate autophagy and abrogated the suppression of IL- $1 \beta$ induced IL- 6 expression. Conclusion: Our findings demonstrate for the first time that Butein activate autophagy in OA chondrocytes via AMPK/TSC2/ULK1/mTOR pathway. Additionally, activation of autophagy
\end{abstract}


was essential to block the IL-1 $\beta$-induced expression of IL- 6 in OA chondrocytes. These data support further studies to evaluate the use of Butein or compounds derived from it for the management of $O A$.

\section{Introduction}

Osteoarthritis (OA) occurs due to irreversible degeneration of articular cartilage, remodeling of subchondral bone, and progressive pain and joint dysfunction. OA is a leading cause of disability in adults resulting in a severe burden on the affected individuals, their families and the society. Development of OA depends on genetic predisposition, physical activity, health status and age. Aging-related OA is symptomatic in at least $30 \%$ of senior citizens, and major trauma to joints leads to the development of post-traumatic $\mathrm{OA}$ relatively quickly, even in young adults. The prevalence of $\mathrm{OA}$ is on the rise worldwide due to steady increase in life expectancy and obesity rates. Recent studies have shown that inflammation plays a key role in the development and pathogenesis of osteoarthritis wherein both the chondrocytes and synovium produce proinflammatory mediators in the joint [1] including the high level expression of IL-6 [2]. Increased expression of inflammatory cytokines and chemokines cause aberrant changes in differentiated chondrocytes function which leads to an excess of chondrocyte catabolic activity, mediated by factors including matrix metalloproteinases (MMPs) and aggrecanases [3]. No current medical treatments are available to prevent progressive joint deterioration and disability characteristic of $\mathrm{OA}$ leaving total joint arthroplasty, which requires major surgery and involves artificial implants that seldom lasts for $>15$ years, as the only viable option. It is thus crucial to find and test compounds with little or no toxicity to prevent and block joint deterioration in people with symptomatic $\mathrm{OA}$ or at high risk for the development of OA, e.g. due to trauma.

Autophagy, a defensive cellular pathway involved in the degradation and recycling of molecular aggregates and dysfunctional cellular organelles, is a key player in the maintenance of cellular homeostasis [4]. Autophagy is upregulated during stress conditions (starvation, oxidative stress, inflammation, etc) and functions as a critical regulator of cellular metabolism [4]. Dysregulation of autophagy has been associated with the pathogenesis of multiple human diseases [5-7]. We and others have shown that autophagy is downregulated in OA cartilage and in IL-1 $\beta$ treated chondrocytes $[8,9]$. Decline in autophagy disrupts the clearance of dysfunctional mitochondria and enhances oxidative stress, inflammation and apoptosis [10]. Autophagy inhibition in chondrocytes resulted in upregulation of cartilage extracellular matrix degrading proteases leading to the development of OA [11,12]. Studies have demonstrated that inhibition of mammalian target of rapamycin (mTOR) pathway acts as a protective mechanism against the development and progression of $\mathrm{OA}[13,14]$. Activation of AMP activated protein kinase $\alpha(A M P K \alpha)$, a positive regulator of autophagy, by metformin has been found to suppress the progression of inflammatory arthritis [15].

Recent studies on traditional medicines have shown that many nutraceuticals have antiinflammatory activities and are good candidate for the prevention and or treatment of $\mathrm{OA}$ [16-18]. Butein (2',3, 4,4'-Tetrahydroxychalcone), a polyphenol produced by several plant species, has been reported to have anti-inflammatory activity in several systems [19-21]. Butein increased the activity of SIRT1 and enhanced the life span of Saccharomyces cerevisiae [22]. Butein has been shown to have anti-cancer activity through the inhibition of Akt/mTOR activity [23]. Butein has also been shown to subdue the proinflammatory effects of IL-1 $\beta$ on chondrocytes [20] however, the mechanism was not reported and largely remains unknown. We hypothesized that Butein exert the anti-inflammatory effects via autophagy activation in OA chondrocytes. To test our hypothesis, we treated human chondrocytes with Butein and studied autophagy activation, mechanism of autophagy activation and its effect on the regulation of IL-6 expression under pathological conditions. Our results demonstrated that Butein activated autophagy in human chondrocytes through AMPK $\alpha / T S C 2 / U L K 1 / m T O R$ pathway. Furthermore, suppression of IL-1 $\beta$-induced expression of IL-6 by Butein was 
dependent on the activation of autophagy as the suppressive effect was completely lost with the inhibition of autophagy. These results identify activation of autophagy via AMPK $\alpha$ TSC2/ ULK1/mTOR pathway by Butein as a critical event in the suppression of IL-6 expression in human chondrocytes under pathological conditions. These data also support further studies on the evaluation of Butein or compounds derived from it as potential therapeutic agents for the treatment and/or prevention of $\mathrm{OA}$.

\section{Materials and Methods}

\section{Reagents and antibodies}

Butein was procured from Extrasynthese (\#1103 S, Extrasynthese, France). The culture media (DMEM-F12) was procured from Lonza (\#12-7192, Walkersville, MD). Pronase and collagenase were from Roche Diagnostics (\#11459643001 and \#11088793001 respectively). TaqMan assays for IL-6 and $\beta$-Actin were from Integrated DNA Technologies (IDT, Coralville, Iowa). Validated antibodies against human IL-6 (\#SC-130326) and $\beta$-Actin (\#SC-47778) were from Santa Cruz Biotechnology (Santa Cruz, CA). Antibodies against MAP1LC3B (\#2775S), P-mTOR (\#5536S), mTOR (\#2983S), P-AMPK $\alpha$ (\#2535S) AMPK $\alpha$ (\#2603S), P-ULK1 (\#12753) and ULK1 (\#8054) were from Cell Signaling Technology (CST, Beverly, MA). Lysotracker Red (\#L7528) for lysosomes staining was obtained from Life Technologies.

\section{Chondrocytes preparation and maintenance}

The study protocol to use discarded, de-identified human cartilage tissue was reviewed and approved by the Institutional Review Board (IRB) of Northeast Ohio Medical University, Rootstown, Ohio as a "nonhuman subject study under $45 \mathrm{CFR}$ ". OA cartilage was from donors who underwent total knee arthroplasty (TKA) and normal cartilage was from donors with no known history of arthritic disease and was obtained from NDRI (National Disease Research Interchange, PA). Chondrocytes were prepared by sequential digestion with Pronase and Collagenase and maintained in DMEM-F12 as described previously [10].

\section{Histopathological analysis of cartilage tissue}

Cartilage tissue was obtained from donors after the knee replacement surgery. The damaged and undamaged areas of cartilage were identified by India ink staining, full thickness cartilage pieces were resected and fixed with $4 \%$ paraformaldehyde. The fixed tissues were dehydrated by passing through a graded series of ethanol (50\%, 70\%, 90\% and 100\%) followed by Xylene wash and embedded in paraffin and $5 \mu \mathrm{M}$ thick sections were cut and stained with Safranin 0/Fast Green protocol. For immunohistochemistry, the tissue sections were deparaffinized in Xylene for 5 minutes and rehydrated by passing through a series of graded ethanol $(100 \%, 90 \%, 70 \%$ and $50 \%)$ as above and finally washed with 1 X-TBS for 10 minutes, and antigen retrieval was performed in $10 \mathrm{mM}$ citrate buffer $(\mathrm{pH}$ 6.0) using a microwave oven for 3 minutes at $700 \mathrm{~W}$. After cooling, sections were washed with 1 XTBS and endogenous peroxidase activity was neutralized by $3 \%$ hydrogen peroxide in 1 XTBS. Sections were washed again with TBS and blocked in $5 \%$ Goat serum for 30 minutes at room temperature and incubated in primary antibody for overnight at $4{ }^{\circ} \mathrm{C}$. Sections were washed with 1XTBS and incubated with HRP conjugated secondary antibody for $1 \mathrm{hr}$ followed by washing and developing with DAB substrate kit (\#34002, Pierce).

\section{Treatment of chondrocytes with IL-1 $\beta$ and Butein}

Normal and OA Chondrocytes were seeded in 6 wells plates $\left(1 \times 10^{6}\right.$ per well) in complete media (DMEM-F12 supplemented with 10\% fetal calf serum) and cultured for $48-72 \mathrm{hr}$ and were then pretreated with $10 \mu \mathrm{g} / \mathrm{ml}(36 \mu \mathrm{M})$ or different concentrations of Butein for $2 \mathrm{hr}$ (or for the indicated time) followed by stimulation with IL-1 $\beta(1 \mathrm{ng} / \mathrm{ml}) .0 .1 \%$ DMSO alone treatment of chondrocytes served as control.

\section{Chondrocyte viability assay}

OA chondrocytes were seeded in 96 well plates $(20,000$ cells/well) and treated with different concentration of Butein for $24 \mathrm{hr}$ or with $10 \mu \mathrm{g} / \mathrm{ml}(36 \mu \mathrm{M})$ of Butein for up to $72 \mathrm{hr}$. The viability of Butein treated chondrocytes was determined by MTT assay as described previously [24]. 


\section{Cellular Physiology Cell Physiol Biochem 2018;49:932-946

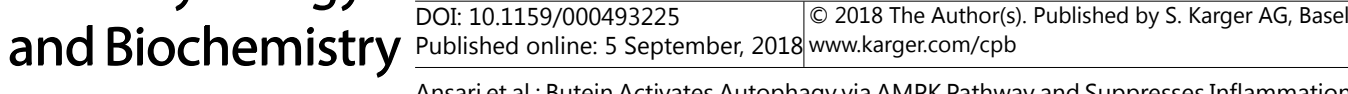

Measurement of autophagy and autophagy flux

Autophagy activation in Butein treated OA chondrocytes was measured by immunoblot analysis of the levels of MAP1LCB-II and the formation of autophagosomes by immunofluorescence microscopy as described previously [8]. Autophagy activation by Butein was determined by taking the ratio of MAP1LC3BII to $\beta$-actin using Image J, as the ratio of MAP1LC3B-II to MAPLC3B-I may not be appropriate measurement of autophagy activation [25]. The number of autophagosomes were quantified by counting the LC3 positive (green) puncta in around 50 chondrocytes in control and Butein treated chondrocytes using Imgae J. Autophagy flux was measured by treating the $\mathrm{OA}$ chondrocytes with Butein overnight followed by treatment with Bafilomycin for $2 \mathrm{hr}$ before lysate preparation and Western blotting to measure the levels of MAP1LCBII protein. The fusion of the autophagosomes with lysosomes represents the last step of the autophagy pathway. We confirmed the fusion of autophagosomes with lysosomes in Butein treated OA chondrocytes by immunofluorescent staining and confocal microscopy using the Olympus FV1000 confocal microscope as described previously [10]. In brief, chondrocytes were seeded in 8 well chamber slides and treated with Butein or DMSO alone as control and fixed with $4 \%$ paraformaldehyde and stained for MAP1LC3B. For lysosomal staining, Lysotracker Red was used to stain the cells for 30 minutes before the end of the experiment.

Determination of ROS levels

ROS levels in OA chondrocytes were measured by DHR123 or DCFDA staining followed by flow cytometry as described previously [8]. In brief, OA chondrocytes were stained with DCFDA or DHR123 for 30 minutes followed by treatment with Butein for 5 minutes, washed with PBS and analyzed for DCFDA or DHR123 fluorescence by flow cytometer (BD Accuri C6) and the data was analyzed by FlowJo software.

\section{Western blotting and ELISA of IL-6}

Normal or OA chondrocytes treated with Butein or IL-1 $\beta$ were lysed in RIPA buffer supplemented with phosphatase and protease inhibitors cocktail. The lysate $(20 \mu \mathrm{g})$ was resolved on $10 \%$ or $12 \%$ SDSPAGE and Western blotting with validated antibodies was done as described previously [26]. Chondrocytes stimulated with IL-1 $\beta$ secrete very high amount of IL- 6 in the culture supernatant that can be analyzed by ELISA as well as Western blotting [2]. So, we collected the culture supernatants from Butein and/or IL-1 $\beta$ treated chondrocytes and determined the levels of IL-6 by ELISA or equal volume $(30 \mu \mathrm{l})$ was used for Western blotting to determine the secreted levels of IL-6.

\section{RNA isolation and TaqMan Assay}

Normal or OA chondrocytes seeded in 6 well plate $\left(1 \times 10^{6}\right.$ cells/well) were treated with IL- $1 \beta(1 \mathrm{ng} /$ $\mathrm{ml}$ ) or Butein $(10 \mu \mathrm{g} / \mathrm{ml}$ or $36 \mu \mathrm{M})$ for $2 \mathrm{hrs}$ followed by IL- $1 \beta(1 \mathrm{ng} / \mathrm{ml})$ treatment for overnight. Total RNA was isolated using RNeasy kit (\#74104, Qiagen) and gene expression was quantified using TaqMan assay as described [10].

siRNA transfection

siRNA for ATG5 knockdown was procured from Qiagen (\#GS9474). OA chondrocytes $(70 \%$ confluent) were transfected with siATG5 or Control siRNA using X-tremeGENE siRNA transfection reagent (\#4476093001, Roche). siATG5 or siControl transfected chondrocytes were pretreated with Butein for 2 hrs followed by stimulation with IL-1 $\beta$. siRNA-mediated knockdown of ATG5 expression was confirmed by Western blotting using a validated antibody (\#12994, CST). Effect of ATG5 depletion on autophagy was assessed by immunofluorescence staining of autophagosomes with MAP1LC3B antibody (\#2775S, CST) or the levels of MAP1LC3B-II formation by Western blotting. The effect of ATG5 knockdown on Butein mediated suppression of IL- 6 was assessed by pretreating siControl or siATG5 transfected chondrocytes with Butein followed by stimulation with IL-1 $\beta$. The chondrocytes were harvested for total RNA preparation using a commercially available kit (\#74104, Qiagen) that was used subsequently for cDNA preparation and quantification of IL-6 mRNA levels by TaqMan assay. 


\section{Cellular Physiology

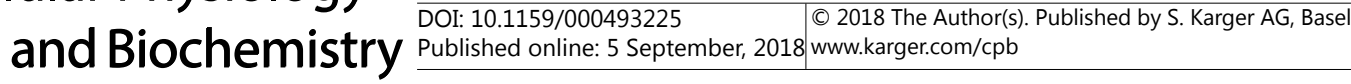

Ansari et al.: Butein Activates Autophagy via AMPK Pathway and Suppresses Inflammation

\section{Statistical analysis}

All the data were analyzed for statistical significance using the software SigmaPlot version 12.3 (Systat Software Inc.) Values are represented as Mean \pm SD. The statistical significance between two groups were analyzed by t-test whereas statistical significance between experimental and control groups were determined by using one-way analysis of variance (ANOVA) followed by Tukey's test for post hoc analysis. Each experiment was repeated at least three times using chondrocytes from three independent patient samples. P value $<0.05$ was considered as statistically significant.

\section{Results}

IL-6 was highly expressed in the damaged areas of OA cartilage and in IL-1 $\beta$ treated chondrocytes

OA joints have high levels of proinflammatory cytokines, chemokines and prostaglandin levels $[1,27]$. To measure the levels of IL-6, we isolated cartilage tissue pieces from smooth (unfibrillated or undamaged) and damaged (fibrillated) areas of cartilage from OA donors and analyzed for proteoglycan levels by Safranin O-fast green staining. In comparison to smooth cartilage, the cartilage from damaged area showed significant loss of proteoglycan levels (Fig. 1A, left panel). Immunohistochemistry of IL-6 on cartilage sections showed high levels of IL-6 expression in the damaged areas, in comparison to undamaged areas (Fig. 1A, right panel). Proinflammatory cytokine IL-1 $\beta$ is expressed at high levels in OA joints and treatment of chondrocytes with recombinant human IL-1 $\beta$ induces the expression of $\mathrm{OA}$ signature genes $[28,29]$. Analysis of IL-6 mRNA and protein levels showed upregulation of IL-6 expression in IL-1 $\beta$ stimulated OA chondrocytes (Fig. 1B and 1C, respectively). These results showed that OA joints have high levels of IL- 6 expression and IL-1 $\beta$ treatment of chondrocytes increased the expression of IL- 6 mimicking the pathological conditions of OA.

Fig. 1. IL-6 is highly expressed in the damaged areas of $\mathrm{OA}$ joints. (A) Cartilage pieces were taken from the damaged and smooth areas of cartilage obtained from OA donors and processed for histopathology. The cartilage sections were analyzed by Safranin $\mathrm{O}$-fast green staining to determine the level of degradation of proteoglycan (left panel) and immunohistochemistry to determine the levels of IL-6 (right panel). (B) Chondrocytes were isolated from $\mathrm{OA}$ donors and maintained in DMEM/F12 supplemented with $10 \%$ serum. Chondrocytes were left untreated (control) or treated with IL-1 $\beta$ ( $1 \mathrm{ng} / \mathrm{ml}$ ) for overnight (16 hrs) and cells were harvested for total RNA preparation and the mRNA levels of IL-6 was analyzed by RT-qPCR using TaqMan assays. $\beta$-Actin was used as normalization control, $\left({ }^{*} \mathrm{P}<0.005\right)$. (C) Chondrocytes were treated with IL-1 $\beta$ ( $1 \mathrm{ng} / \mathrm{ml}$ ) for indicated time and the lysate was prepared and analyzed for the IL- 6 expression levels by Western blotting.

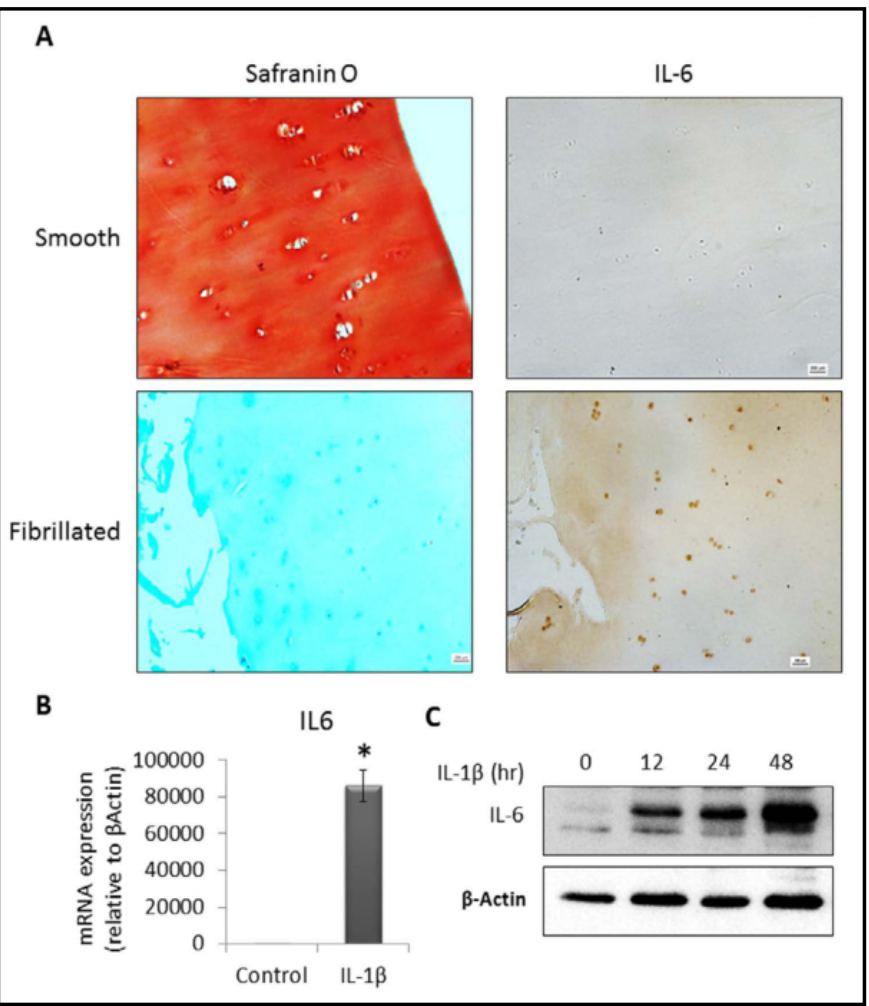




\section{Butein did not affect the viability of $O A$ chondrocytes in vitro}

There are reports that have shown that Butein reduces cell viability and enhances apoptosis in multiple cancer cells including ovarian cancer cells [30] cervical cancer cells [23], neuroblastoma [31] and T-cell lymphoma [32]. In order to study the effects of Butein on the viability of $\mathrm{OA}$ chondrocytes, $\mathrm{OA}$ chondrocytes were treated with different concentrations of Butein [0.6 $\mu \mathrm{g} / \mathrm{ml}-10 \mu \mathrm{g} / \mathrm{ml}$ (or $2.25 \mu \mathrm{M}-36 \mu \mathrm{M}$ )] for $24 \mathrm{hr}$ or with $10 \mu \mathrm{g} / \mathrm{ml}$ Butein for up to $72 \mathrm{hr}$ and the viability was analyzed by MTT assay. The results showed no significant toxic effects of Butein on the viability of primary human OA chondrocytes in vitro (Fig. 2A and 2B). Based on this data we conclude that Butein was non-toxic to human OA chondrocytes in vitro in the dose range tested.

\section{Butein inhibited the $I L-1 \beta$ induced expression of IL-6 in normal and OA chondrocytes}

In order to study the anti-inflammatory effect of Butein on chondrocytes under pathological conditions, we used chondrocytes isolated from donors with no known history of $\mathrm{OA}$ and treated with IL-1 $\beta$ to mimic pathological conditions which has been shown by us and others to induce the expression of OA signature genes $[8,28]$. We pretreated the chondrocytes with Butein $(10 \mu \mathrm{g} / \mathrm{ml})$ for $2 \mathrm{hr}(0.1 \%$ DMSO alone was used as control) followed by IL-1 $\beta(1 \mathrm{ng} / \mathrm{ml})$ treatment for $16 \mathrm{hr}$ and harvested for either RNA isolation or cell lysate preparation to analyze the levels of IL- 6 mRNA and protein expression respectively. Stimulation of normal human chondrocytes with IL-1 $(1 \mathrm{ng} / \mathrm{ml})$ increased the expression of IL-6 mRNA (Fig. 2C) and protein levels (Fig. 2D) significantly compared to controls $(\mathrm{P}<0.005)$. Importantly, pretreatment of chondrocytes with Butein dramatically suppressed the IL- $1 \beta$ induced expression of IL- 6 at mRNA and protein levels (Fig. $2 \mathrm{C}$ and 2D respectively, $\mathrm{P}<0.05$ ). We observed similar results with chondrocytes isolated from donors with OA (Fig. 2E and $2 \mathrm{~F}$ ). To rule out that this effect was due to the possible dedifferentiation of chondrocytes in monolayer culture, we prepared cartilage explants from the undamaged areas of OA cartilage and treated them with Butein and IL- $1 \beta$ as above. The level of IL- 6 protein in the cartilage explants was then analyzed by immunohistochemistry and in the culture supernatant by ELISA and immunoblotting. IL-1 $\beta$ stimulation resulted in increased expression of IL-6 protein in human cartilage explants which was suppressed by pre-treatment with Butein (Fig. 2G, 2H and 2I). These results demonstrated that Butein has anti-inflammatory property and suppresses the IL- $1 \beta$ induced expression of IL- 6 in human normal and OA chondrocytes and $\mathrm{OA}$ cartilage explants under pathological conditions.

\section{Butein suppressed the IL-6 expression by inducing autophagy}

Autophagy is a protective mechanism against cellular stress and inflammation and OA pathogenesis $[4,11]$. We investigated whether (1) Butein activates autophagy in chondrocytes; and (2) whether Butein-mediated suppression of IL-1 $\beta$ induced expression of IL-6 was via autophagy activation in human chondrocytes. To investigate the first question, $\mathrm{OA}$ chondrocytes were treated with Butein for overnight and analyzed for the expression levels of MAP1LC3B-II protein, a known marker of autophagy activation [25]. Treatment of OA chondrocytes with Butein increased the levels of MAP1LC3B-II protein in a dose dependent manner indicating the activation of autophagy (Fig. 3A and 3B). We further confirmed the activation of autophagy by immunofluorescence staining of endogenous MAP1LC3B protein followed by quantification of autophagosomes. This analysis demonstrated that the number of autophagosomes was significantly increased in OA chondrocytes treated with Butein (Fig. 3C and 3D). Furthermore, we measured the autophagy flux by treating OA chondrocytes with Butein for overnight followed by treatment with Bafilomycin-A1 for $2 \mathrm{hr}$ [33] and measured the levels of MAP1LC3B-II protein by immunoblotting. The increase in MAP1LC3B-II levels by Butein treatment in OA chondrocytes was further increased by Bafilomycin-A1 treatment of OA chondrocytes (Fig. 3E) indicating that the treatment of OA chondrocytes with Butein increased the autophagy flux. We also found that the colocalization of autophagosomes (stained with MAP1LC3B antibody, green color) with lysosomes (stained with Lysotracker 
Fig. 2. Butein is not toxic and inhibits IL$1 \beta$ induced expression of IL-6 in normal and OA chondrocytes. Chondrocytes were seeded in 6-well plates $\left(1 \times 10^{6}\right.$ cells $/$ well) and treated with (A) different concentrations of Butein (36 to $2.25 \mu \mathrm{M}$ or 10 to $0.6 \mu \mathrm{g} / \mathrm{ml}$ ) for 24 hours and (B) $10 \mu \mathrm{g} / \mathrm{ml}$ (or $36 \mu \mathrm{M}$ ) for 24, 48 and $72 \mathrm{hrs}$. DMSO alone (0.1\%) was used as control. Chondrocytes viability was measured by MTT assay. (C) and (D) Chondrocytes prepared from normal donors (no known history of any rheumatic disease) were pretreated with Butein $(10 \mu \mathrm{g} /$ $\mathrm{ml}$ or $36 \mu \mathrm{M}$ ) for 2 hrs followed by addition of IL-1 $\beta$ (1 $\mathrm{ng} / \mathrm{ml}$ ) for overnight. Chondrocytes were harvested either for RNA isolation to determine the expression levels of IL-6 mRNA or cell lysate preparation and immunoblotting of IL-6 $(* \mathrm{P}<0.005, \quad * * \mathrm{P}<0.05)$.

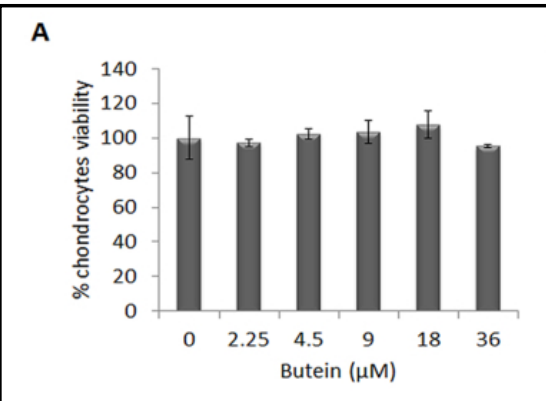

C

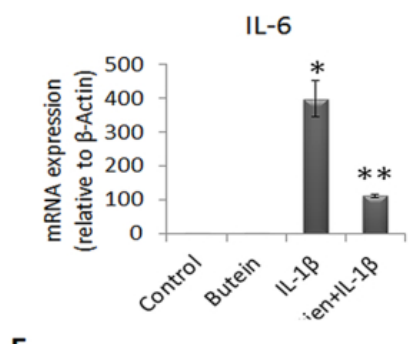

E
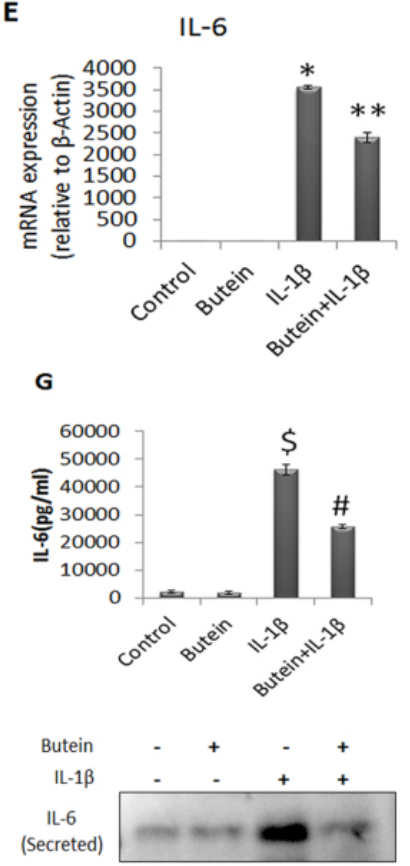

H

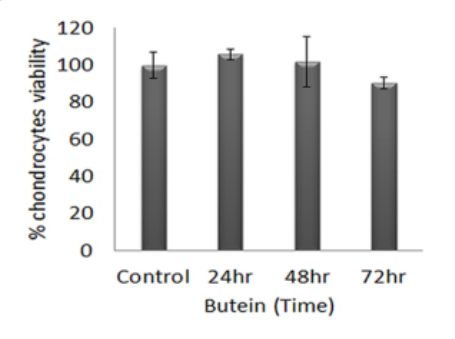

D
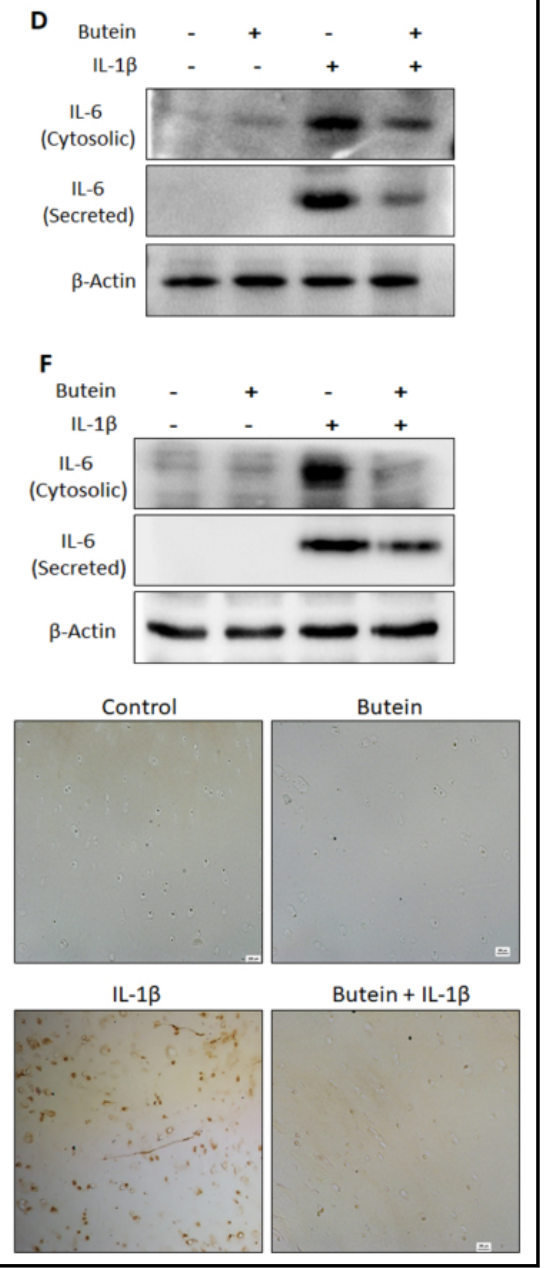

Culture supernatants

were also collected and equal volume of it was loaded on 12\% SDS-PAGE to determine the levels of IL-6 secreted in the culture supernatant. $\beta$-Actin was used as loading or normalization control. (E) and (F) Chondrocytes were isolated from OA donors and treated as above with Butein and IL-1 $\beta$ to measure the levels of IL- 6 mRNA and protein by qPCR and Western blotting. (G) The IL-6 levels in the culture supernatant were determined by ELISA ( $\$ \mathrm{P}<0.05$ vs Control, $\# \mathrm{P}<0.05$ vs $\mathrm{IL}-1 \beta)$. $(\mathrm{H})$ Cartilage explants were prepared from the undamaged areas of cartilage obtained from OA donors and maintained in DMEM/F12 supplemented with $10 \%$ serum. The explants were treated with IL-1 $\beta(10 \mathrm{ng} / \mathrm{ml})$ for $72 \mathrm{hrs}$ in the presence or absence of Butein $(10 \mu \mathrm{g} / \mathrm{ml}$ or $36 \mu \mathrm{M})$. The explants were fixed with $4 \%$ PFA and processed for histological analysis of IL-6 expression levels. (I) The culture supernatant from the above experiment was analyzed for secretory levels of IL- 6 by Western blotting. 


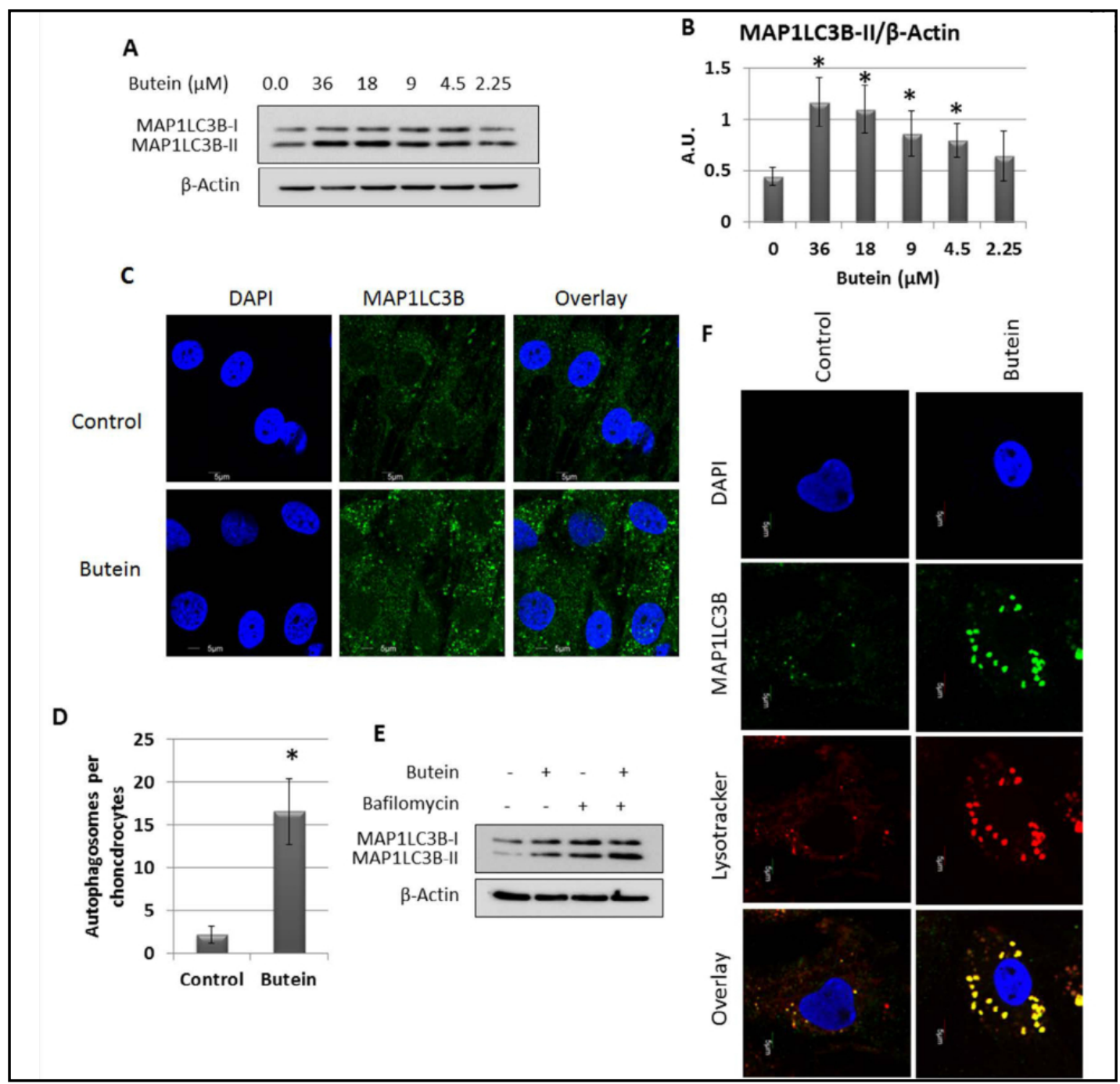

Fig. 3. Butein induced autophagy in chondrocytes. (A) Chondrocytes were treated with different concentrations of Butein for overnight and harvested for cell lysate preparation for immunoblot analysis of MAP1LC3B-II levels. $\beta$-Actin was used as loading control. (B) Bar graph represents the quantification of endogenous MAP1LC3B-II with respect to $\beta$-Actin. (C) OA chondrocytes seeded in 8 well chamber slides were treated with Butein $(10 \mu \mathrm{g} / \mathrm{ml}$ or $36 \mu \mathrm{M})$ for overnight and fixed with $4 \%$ paraformaldehyde and probed for autophagosomes using anti-MAP1LC3B antibody followed by anti-rabbit Alexa-fluor-488 secondary antibody. Chondrocytes treated with $0.1 \%$ DMSO were taken as control. (D) Bar graph represents the quantification of autophagosomes. (E) OA chondrocytes were treated with Butein for overnight (16 hrs) followed by Bafilomycin A1 for 2 hrs to analyze the autophagy flux. Chondrocytes were harvested for cell lysate preparation and measured the levels of MAP1LC3B-II by immunoblotting. $\beta$-Actin was used as loading control. (F) OA chondrocytes seeded in 8 well chamber slides were treated with Butein for overnight followed by the addition of Lysotracer Red for 30 minutes. Chondrocytes were fixed with $4 \%$ paraformaldehyde and probed for autophagosomes using anti-MAP1LC3B antibody followed by anti-rabbit Alexa-fluor-488 secondary antibody. Chondrocytes treated with DMSO were taken as control. DAPI was used to counterstain the nuclei $(* \mathrm{P}<0.05)$.

Red, red color) was increased in Butein treated OA chondrocytes (Fig. 3F) demonstrating the maturation of the response.

In order to test the hypothesis that autophagy activation by Butein is required to suppress the IL-1 $\beta$ induced expression of IL- 6 in human OA chondrocytes, we suppressed autophagy using siRNA mediated knockdown of autophagy related protein 5 (ATG5) and autophagy 


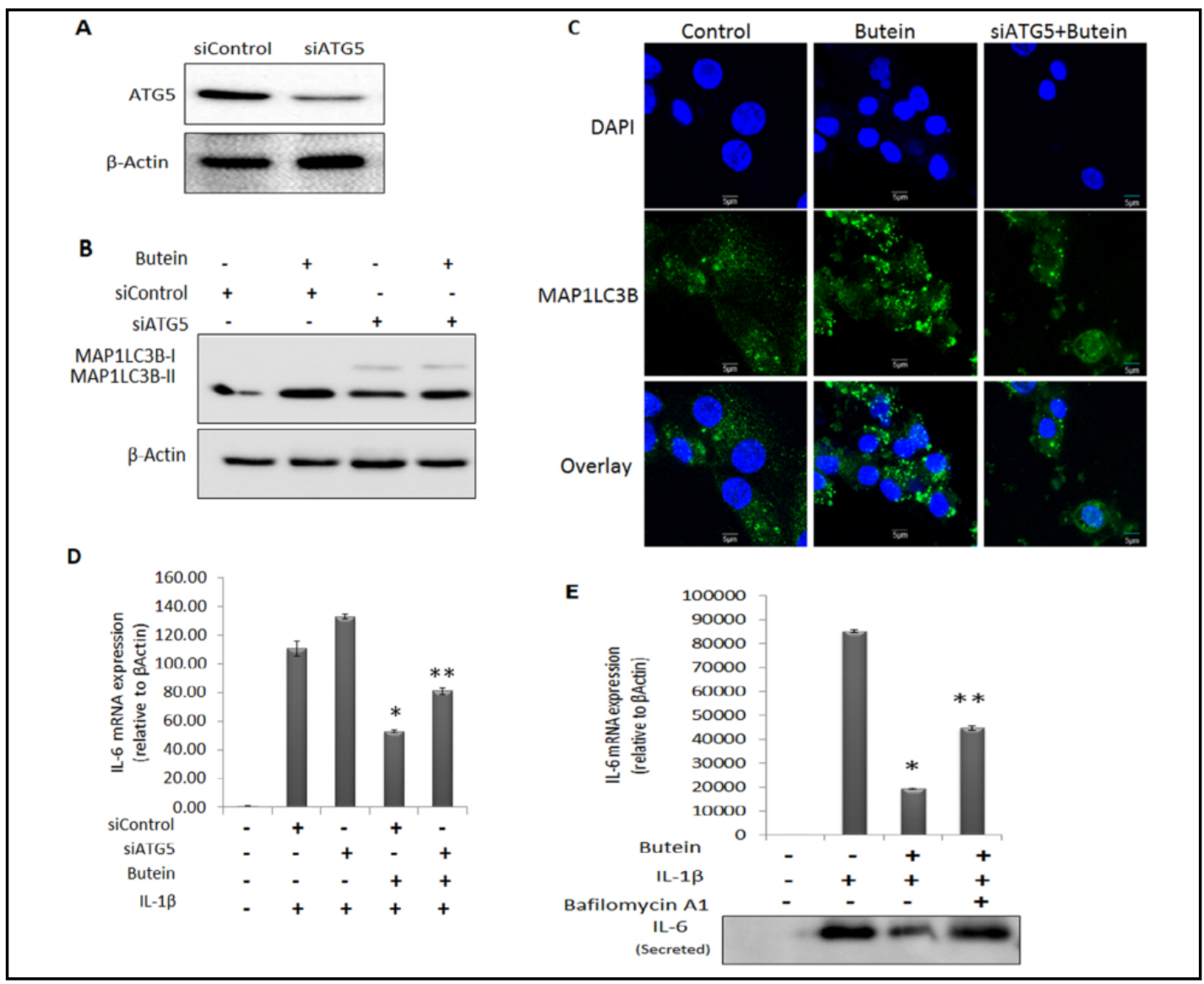

Fig. 4. Butein mediated suppression of IL-6 expression was dependent on autophagy. (A) Chondrocytes were transfected with siRNA targetting ATG5 (siATG5) or scrambled control (siControl) for 48 hrs. ATG5 knockdown was confirmed by Western blotting. (B) siATG5 or siControl transfected chondrocytes were treated with Butein $(10 \mu \mathrm{g} / \mathrm{ml}$ or $36 \mu \mathrm{M})$ and harvested to prepare lysate and MAP1LC3B-II levels were determined by immunoblotting. $\beta$-Actin was used as loading control. (C) Chondrocytes were transfected as above and treated with Butein to analyze the formation of autophagosomes by immunofluorescence staining of MAP1LC3B. DAPI was used to counterstain the nuclei. (D) The chondrocytes were transfected as above and pretreated with Butein followed by IL-1 $\beta$. The chondrocytes were harvested for RNA preparation and IL-6 mRNA levels were determined by qPCR. $\beta$-Actin was used as normalization control $\left(* \mathrm{P}<0.05\right.$, $\left.{ }^{* *} \mathrm{P}<0.01\right)$. (E) chondrocytes were pretreated with autophagy inhibitor Bafilomycin for $2 \mathrm{hrs}$ followed by Butein $(10 \mu \mathrm{g} /$ $\mathrm{ml}$ or $36 \mu \mathrm{M}$ ) and IL-1 $\beta$. The chondrocytes were used to prepare total RNA for the analysis of IL-6 mRNA and culture supernatant was used to determine the levels of IL- 6 protein. $\beta$-Actin was used as normalization control $(* \mathrm{P}<0.05, * * \mathrm{P}<0.01)$.

inhibitor Bafilomycin-A1. ATG5 is essential for autophagy process and is required for the formation of autophagosomes [34]. We knocked down the ATG5 expression in chondrocytes using siRNA (Fig. 4A) and analyzed the effect of Butein on autophagy activation. ATG5 depletion markedly reduced the levels of Butein induced MAP1LC3B-II in OA chondrocytes (Fig. 4B). Additionally the numbers of autophagosomes in ATG5 depleted chondrocytes were also reduced (Fig. 4C). Of importance is our finding that the Butein mediated suppression of IL-1 $\beta$ induced IL-6 expression was inhibited by ATG5 depletion (Fig. 4D) further confirming that active autophagy was required for the anti-inflammatory activity of Butein in human OA chondrocytes. Further, human OA chondrocytes were pretreated with Bafilomycin-A1 to block autophagy and then treated with Butein followed by IL-1 $\beta$ treatment and the expression of IL- 6 was analyzed. Butein mediated suppression of IL- $1 \beta$ induced IL- 6 expression in OA chondrocytes was abrogated by pre-treatment with Bafilomycin-A1 (Fig. 4E) indicating that 
Fig. 5. Butein inhibited mTOR pathway in OA chondrocytes. To measure the levels of ROS in response to Butein treatment, chondrocytes were stained with DCFDA (A) or DHR123 (B) followed by addition of Butein $(10 \mu \mathrm{g} / \mathrm{ml}$ or $36 \mu \mathrm{M})$ for 5 minutes. Chondrocytes were analyzed by flow cytometer for DCFDA or DHR123 fluorescence $\quad\left({ }^{*} \mathrm{P}<0.05\right)$. (C) Chondrocytes were treated with Butein for indicated time and harvested for lysate preparation followed by immunoblot analysis of Phospho(Ser2448)mTOR and t-mTOR, Phospho(S er 411 ) p7056K and $\beta$-Actin. (D)

Represents densitometric analysis of P-mTOR/mTOR $\left({ }^{*} \mathrm{P}<0.05\right)$. (E) Chondrocytes were seeded in 8 well chamber slides and treated with Butein for $2 \mathrm{hrs}$. Chondrocytes were fixed in $4 \%$ paraformaldehyde and probed for mTOR and LAMP1 to analyze the colocalization of mTOR with lysosomes. DAPI was used to counterstain nuclei.

the suppression of IL-6 expression by Butein was dependent on active autophagy in human OA chondrocytes. Taken together these results demonstrate that Butein-mediated activation of autophagy is a critical event that is required for the suppression of IL-6 expression in human OA chondrocytes under pathological conditions.

Butein activated autophagy via AMPK/TSC2/ULK1/mTOR pathwayin human chondrocytes

Reactive Oxygen Species (ROS) has been reported to activate autophagy in other cell types [35]. Butein has been shown to increase ROS levels in neuroblastoma cells and breast cancer cells $[31,36]$ and also functions as antioxidant for liver stellate cells and keratinocytes $[21,37]$. OA chondrocytes treated with Butein showed no increase in ROS levels as measured by DCFDA (Fig. 5A) and DHR123 staining (Fig. 5B), rather we found a significant decrease in the ROS levels in OA chondrocytes treated suggesting that Butein acts as an antioxidant in primary OA chondrocytes.

Activation of mammalian target of rapamycin (mTOR) signaling pathway is a well-known negative regulator of autophagy activation; therefore, we investigated the effect of Butein treatment on mTOR activation in human OA chondrocytes. The phosphorylation of mTOR Ser-2448, which is a marker for its activity [38], was determined by Western immunoblotting and it was found that the phosphorylation of mTOR ${ }^{\text {Ser-2448 }}$ was decreased in human OA chondrocytes treated with Butein (Fig. 5C and 5D). We further confirmed Butein-mediated inhibition of mTOR activity by measuring the phosphorylation of its downstream target p70S6 kinase. Phosphorylation of p70S6 kinase was negligible (Fig. 5C) in OA chondrocytes treated with Butein confirming suppression of mTOR activity in human OA chondrocytes treated with Butein. Furthermore, as mTOR activity requires the association of mTORC1 
Fig. 6. Butein activated AMPK $\alpha / T S C 2 / U L K 1 \quad$ pathway in chondrocytes to activate autophagy. (A) Chondrocytes were treated with Butein for indicted time and the phosphorylation levels of Phospho(Thr-172)AMPKo, Phospho(S1387)-TSC2 and Phospho(S317)-ULK1 were determined by Western blotting. $\beta$-Actin and respective total levels of proteins were used as normalization control. (B) Bar graph represents the densitometric analysis of Western blots from at least three different samples $\left({ }^{*} \mathrm{P}<0.05\right)$.

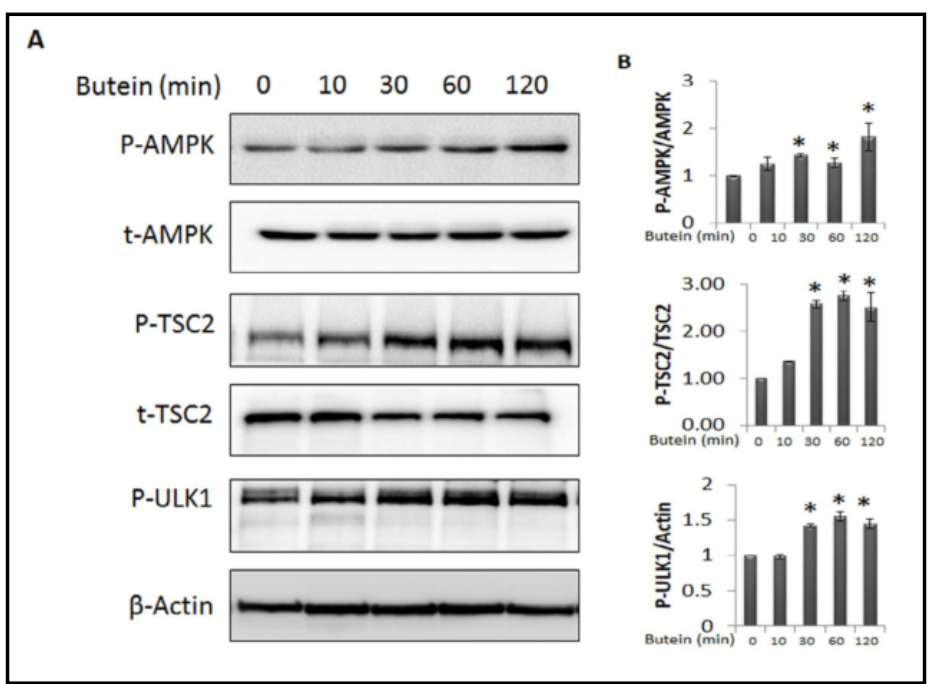

complex to the lysosomal surface [39], we investigated the colocalization of mTOR with lysosomes by confocal microscopy using LAMP1 as lysosomal marker. In control cultures, mTOR staining showed punctate pattern which were colocalized with lysosomes. However, Butein treated OA chondrocytes showed diffused staining of mTOR suggesting that Butein treatment displaced the mTOR from the surface of lysosomes (Fig. 5E).

AMPK $\alpha$ is the energy sensor and functions as positive regulator of autophagy via phosphorylation based activation of ULK1 and inhibition of MTOR via TSC2 [40]. First we investigated the activation of AMPK $\alpha$ in OA chondrocytes upon Butein treatment. OA chondrocytes treated with Butein showed increased phosphorylation of AMPK $\alpha^{\text {Thr-172 }}$ suggestive of AMPK $\alpha$ activation (Fig. 6A and 6B). The activation of AMPK $\alpha$ was further confirmed by monitoring the TSC2 and ULK1 phosphorylation. Butein treatment resulted in increased phosphorylation of TSC2 $2^{\text {Ser-1387 }}$ and ULK1 ${ }^{\text {Ser-317 }}$ compared to controls (Fig. $6 \mathrm{~A}$ and $6 \mathrm{~B}$ ) indicating that Butein treatment activated AMPK $\alpha$ in OA chondrocytes. Taken together these results indicate that Butein activates AMPK $\alpha /$ TSC2/ULK1/ mTOR pathway to activate autophagy which is critical for the suppression of the expression of inflammatory mediators in OA chondrocytes under pathological conditions.
Fig. 7. Schematic diagram depicting the pathway through which Butein activates autophagy and blocks inflammation. Butein activated AMPK which is an activator of ULK1 and TSC2. ULK1 is directly involved in autophagy activation. TSC2 inhibits mTOR via Rheb GTPase eventually leading to autophagy activation. 


\section{Cellular Physiology and Biochemistry

\section{Discussion}

Osteoarthritis is the most common joint disease and a major cause of disability in the affected population. Joint failure caused by arthritis leads to enormous costs to society and, importantly, affects patient quality of life. Recent research has significantly improved our understanding of $\mathrm{OA}$ pathogenesis but still there is no disease modifying drug available and the only viable option is the total knee arthroplasty whose incidence is projected to rise by 2050 to $855 \%$ by volume compared to the incidence reported in 2012 in the US. TKA is expensive, temporary and not without risk, therefore alternative strategies for treatment are needed urgently. Autophagy is a cellular catabolic process that helps in the clearance of protein aggregates and dysfunctional organelles [41]. Aging is associated with decreased autophagy in cartilage causing increased mitochondrial dysfunction, oxidative stress and inflammation in joints eventually leading to the development of OA. Moreover, autophagy is downregulated in $\mathrm{OA}$ cartilage in comparison to normal cartilage tissue [11]. Several studies have demonstrated that activation of autophagy promotes/sustains the activation of anabolic pathways essential for cartilage health and suppresses catabolic pathways involved in OA pathogenesis $[8,12,14,41]$. Autophagy inhibition was shown to enhance cartilage degradation resulting in increased severity of the disease [12]. Another study demonstrated that cartilage specific deletion of mTOR, which activated autophagy in cartilage chondrocytes, suppressed the progression of OA in a mouse model of experimental OA [42]. These findings suggest that autophagy pathway likely represent a promising therapeutic target for the prevention and progression of OA. In the present study we have demonstrated for the first time that Butein activated autophagy in chondrocytes and suppressed the expression of inflammatory mediators in vitro. The fact was further supported by our data that autophagy inhibition by siRNA-mediated depletion of ATG5 abrogated Butein mediated suppression of IL-1 $\beta$ induced expression of IL-6.

Recent work has shown promising effects of Butein against cancer, inflammation and oxidative stress [43]. OA joints have high levels of inflammatory molecules and we show herein that Butein, a plant derived small molecule, suppressed the IL-1 $\beta$ induced expression of inflammatory mediator IL- 6 in human OA chondrocytes. This is in accordance to the previous reports that have shown that Butein has anti-inflammatory activity and suppressed inflammation in a variety of cells including human mast cells, macrophages, HUVEC cells etc $[19,44,45]$. The plant extracts containing Butein (for example from Butea monosperma, Rhus verniciflua, etc.) have been used traditionally in Indian Ayurvedic and Unani and Chinese medicine system for the treatment of various human ailments $[43,46]$.

In this study we explored the mechanism of Butein mediated suppression of IL-1 $\beta$ induced expression of IL-6 in human chondrocytes. In previous studies mTOR activity was found to be increased (which suppresses autophagy) in OA cartilage and deletion of mTOR protected mice form the development of $\mathrm{OA}$ and this observation has directed the attention towards suppression of mTOR activity as a potential therapeutic target [42, 47]. Our finding that Butein functions as a suppressor of mTOR activity opens up the avenue for its further development as a potential therapeutic for the effective management of OA. Although, earlier studies showed the inhibition of mTOR pathway by Butein in cervical cancer and $\mathrm{T}$ cell lymphoma $[23,32]$ but the mechanism of Butein mediated suppression of mTOR activity was not explored. Thus the data presented in this study is distinct and novel as we demonstrate that Butein activate AMPK $\alpha$, which phosphorylated its downstream target TSC2 to displace mTOR from the lysosomes resulting in the suppression of mTOR activity in human chondrocytes. We further analyzed the effect of AMPK $\alpha$ activation on its downstream target, ULK1, which is known to phosphorylate Beclin1 and Vps34 complex [48] and activate autophagy. Butein enhanced AMPK $\alpha$ mediated phosphorylation of ULK1 and activated autophagy and autophagy flux and suppressed the expression of IL-6. AMPK $\alpha$ functions as cellular energy sensor and autophagy regulator and has been drawing increased attention of researchers. AMPK $\alpha$ activity is down modulated in aged mouse cartilage [49] and OA 


\section{Cellular Physiology Cell Physiol Biochem 2018;49:932-946 \\ $\begin{array}{ll}\text { DOI: } 10.1159 / 000493225 & \\ \text { and Biochemistry } 2018 \text { The Author(s). Published by S. Karger AG, Basel }\end{array}$ \\ Ansari et al.: Butein Activates Autophagy via AMPK Pathway and Suppresses Inflammation}

chondrocytes [50] and deletion of AMPK $\alpha$ in chondrocytes resulted in enhanced progression of experimental as well as spontaneous OA [51].

In summary, we demonstrate that Butein activated autophagy via AMPK $\alpha / T S C 2 / U L K 1 /$ mTOR pathway suppressed the expression of IL-6 in human chondrocytes under pathological conditions (Fig. 7). These findings suggest that Butein, or compounds derived from it, may be developed for the effective management of OA.

\section{Acknowledgements}

Research reported in this publication was supported by the NIH/National Institute of Arthritis and Musculoskeletal and Skin Diseases and the NIH/National Center for Complementary and Integrative Health of the National Institutes of Health under Award Numbers 1R01AR067056, and AT007373 respectively. The content is solely the responsibility of the authors and does not necessarily represent the official views of the National Institutes of Health.

MYA, NA and TMH conceived and designed the study. MYA and NA performed the experiments. MYA, NA and TMH analyzed and interpreted the data and wrote the manuscript.

\section{Disclosure Statement}

No conflict of interests exists.

\section{References}

$>1$ Goldring MB, Otero M: Inflammation in osteoarthritis. Curr Opin Rheumatol 2011;23:471-478.

-2 Haseeb A, Ansari MY, Haqqi TM: Harpagoside suppresses IL-6 expression in primary human osteoarthritis chondrocytes. J Orthop Res 2017;35:311-320.

-3 Houard X, Goldring MB, Berenbaum F: Homeostatic mechanisms in articular cartilage and role of inflammation in osteoarthritis. Curr Rheumatol Rep 2013;15:375.

4 Ryter SW, Cloonan SM, Choi AM: Autophagy: a critical regulator of cellular metabolism and homeostasis. Mol Cells 2013;36:7-16.

5 Jiang P, Mizushima N: Autophagy and human diseases. Cell Res 2014;24:69-79.

6 Choi AM, Ryter SW, Levine B: Autophagy in human health and disease. N Engl J Med 2013;368:651-662.

7 Levine B, Kroemer G: Autophagy in the pathogenesis of disease. Cell 2008;132:27-42.

8 Ansari MY, Khan NM, Haqqi TM: A standardized extract of Butea monosperma (Lam.) flowers suppresses the IL-1 $\beta$-induced expression of IL- 6 and matrix-metalloproteases by activating autophagy in human osteoarthritis chondrocytes. Biomed Pharmacother 2017;96:198-207.

-9 Carames B, Olmer M, Kiosses WB, Lotz MK: The relationship of autophagy defects to cartilage damage during joint aging in a mouse model. Arthritis Rheumatol 2015;67:1568-1576.

-10 Ansari MY, Khan NM, Ahmad I, Haqqi TM: Parkin clearance of dysfunctional mitochondria regulates ROS levels and increases survival of human chondrocytes. Osteoarthritis Cartilage 2018;26:1087-1097.

11 Carames B, Taniguchi N, Otsuki S, Blanco FJ, Lotz M: Autophagy is a protective mechanism in normal cartilage, and its aging-related loss is linked with cell death and osteoarthritis. Arthritis Rheum 2010;62:791-801.

12 Sasaki H, Takayama K, Matsushita T, Ishida K, Kubo S, Matsumoto T, Fujita N, Oka S, Kurosaka M, Kuroda R: Autophagy modulates osteoarthritis-related gene expression in human chondrocytes. Arthritis Rheum 2012;64:1920-1928.

-13 Khan NM, Ansari MY, Haqqi TM: Sucrose, But Not Glucose, Blocks IL1-beta-Induced Inflammatory Response in Human Chondrocytes by Inducing Autophagy via AKT/mTOR Pathway. J Cell Biochem 2017;118:629-639. 


\section{Cellular Physiology Cell Physiol Biochem 2018;49:932-946 and Biochemistry DOI: 10.1159/000493225 2018 (O) The Author(s). Published by S. Karger AG, Basel

14 Carames B, Hasegawa A, Taniguchi N, Miyaki S, Blanco FJ, Lotz M: Autophagy activation by rapamycin reduces severity of experimental osteoarthritis. Ann Rheum Dis 2012;71:575-581.

15 Yan H, Zhou HF, Hu Y, Pham CT: Suppression of experimental arthritis through AMP-activated protein kinase activation and autophagy modulation. J Rheum Dis Treat 2015;1:5-14.

-16 Shen CL, Smith BJ, Lo DF, Chyu MC, Dunn DM, Chen CH, Kwun IS: Dietary polyphenols and mechanisms of osteoarthritis. J Nutr Biochem 2012;23:1367-1377.

17 Leong DJ, Choudhury M, Hirsh DM, Hardin JA, Cobelli NJ, Sun HB: Nutraceuticals: potential for chondroprotection and molecular targeting of osteoarthritis. Int J Mol Sci 2013;14:23063-23085.

$>18$ Akhtar N, Haqqi TM: Current nutraceuticals in the management of osteoarthritis: a review. Ther Adv Musculoskelet Dis 2012;4:181-207.

19 Rasheed Z, Akhtar N, Khan A, Khan KA, Haqqi TM: Butrin, isobutrin, and butein from medicinal plant Butea monosperma selectively inhibit nuclear factor-kappaB in activated human mast cells: suppression of tumor necrosis factor-alpha, interleukin (IL)-6, and IL-8. J Pharmacol Exp Ther 2010;333:354-363.

20 Zheng W, Zhang H, Jin Y, Wang Q Chen L, Feng Z, Chen H, Wu Y: Butein inhibits IL-1beta-induced inflammatory response in human osteoarthritis chondrocytes and slows the progression of osteoarthritis in mice. Int Immunopharmacol 2017;42:1-10.

21 Seo WY, Youn GS, Choi SY, Park J: Butein, a tetrahydroxychalcone, suppresses pro-inflammatory responses in HaCaT keratinocytes. BMB Rep 2015;48:495-500.

-22 Howitz KT, Bitterman KJ, Cohen HY, Lamming DW, Lavu S, Wood JG, Zipkin RE, Chung P, Kisielewski A, Zhang LL, Scherer B, Sinclair DA: Small molecule activators of sirtuins extend Saccharomyces cerevisiae lifespan. Nature 2003;425:191-196.

23 Bai X, Ma Y, Zhang G: Butein suppresses cervical cancer growth through the PI3K/AKT/mTOR pathway. Oncol Rep 2015;33:3085-3092.

-24 Khan NM, Haseeb A, Ansari MY, Devarapalli P, Haynie S, Haqqi TM: Wogonin, a plant derived small molecule, exerts potent anti-inflammatory and chondroprotective effects through the activation of ROS/ ERK/Nrf2 signaling pathways in human Osteoarthritis chondrocytes. Free Radic Biol Med 2017;106:288301.

25 Mizushima N, Yoshimori T: How to interpret LC3 immunoblotting. Autophagy 2007;3:542-545.

-26 Khan NM, Haseeb A, Ansari MY, Haqqi TM: A wogonin-rich-fraction of Scutellaria baicalensis root extract exerts chondroprotective effects by suppressing IL-1beta-induced activation of AP-1 in human OA chondrocytes. Sci Rep 2017;7:1-14.

27 Ansari MY, Haqqi TM: Interleukin-1beta induced Stress Granules Sequester COX-2 mRNA and Regulates its Stability and Translation in Human OA Chondrocytes. Sci Rep 2016;6:1-13.

28 Daheshia M, Yao JQ: The interleukin 1beta pathway in the pathogenesis of osteoarthritis. J Rheumatol 2008;35:2306-2312.

29 Kapoor M, Martel-Pelletier J, Lajeunesse D, Pelletier JP, Fahmi H: Role of proinflammatory cytokines in the pathophysiology of osteoarthritis. Nat Rev Rheumatol 2011;7:33-42.

-30 Yang PY, Hu DN, Lin IC, Liu FS: Butein Shows Cytotoxic Effects and Induces Apoptosis in Human Ovarian Cancer Cells. Am J Chin Med 2015;43:769-782.

-31 Chen YH, Yeh CW, Lo HC, Su SL, Hseu YC, Hsu LS: Generation of reactive oxygen species mediates buteininduced apoptosis in neuroblastoma cells. Oncol Rep 2012;27:1233-1237.

-32 Ishikawa C, Senba M, Mori N: Butein inhibits NF-kappaB, AP-1 and Akt activation in adult T-cell leukemia/ lymphoma. Int J Oncol 2017;51:633-643.

-33 Mauvezin C, Neufeld TP: Bafilomycin A1 disrupts autophagic flux by inhibiting both V-ATPase-dependent acidification and Ca-P60A/SERCA-dependent autophagosome-lysosome fusion. Autophagy 2015;11:14371438.

34 Mizushima N, Yoshimori T, Ohsumi Y: The role of Atg proteins in autophagosome formation. Annu Rev Cell Dev Biol 2011;27:107-132.

-35 Scherz-Shouval R, Shvets E, Fass E, Shorer H, Gil L, Elazar Z: Reactive oxygen species are essential for autophagy and specifically regulate the activity of Atg4. EMBO J 2007;26:1749-1760.

-36 Yang LH, Ho YJ, Lin JF, Yeh CW, Kao SH, Hsu LS: Butein inhibits the proliferation of breast cancer cells through generation of reactive oxygen species and modulation of ERK and p38 activities. Mol Med Rep 2012;6:1126-1132. 


\section{Cellular Physiology Cell Physiol Biochem 2018;49:932-946

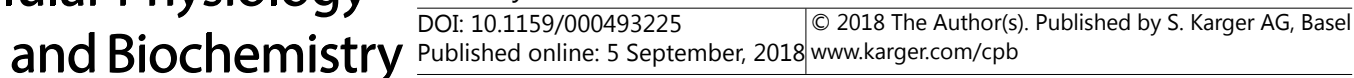 \\ Ansari et al.: Butein Activates Autophagy via AMPK Pathway and Suppresses Inflammation}

37 Szuster-Ciesielska A, Mizerska-Dudka M, Daniluk J, Kandefer-Szerszen M: Butein inhibits ethanol-induced activation of liver stellate cells through TGF-beta, NFkappaB, p38, and JNK signaling pathways and inhibition of oxidative stress. J Gastroenterol 2013;48:222-237.

38 Rosner M, Siegel N, Valli A, Fuchs C, Hengstschlager M: mTOR phosphorylated at S2448 binds to raptor and rictor. Amino Acids 2010;38:223-228.

-39 Sancak Y, Bar-Peled L, Zoncu R, Markhard AL, Nada S, Sabatini DM: Ragulator-Rag complex targets mTORC1 to the lysosomal surface and is necessary for its activation by amino acids. Cell 2010;141:290-303.

-40 Di Nardo A, Wertz MH, Kwiatkowski E, Tsai PT, Leech JD, Greene-Colozzi E, Goto J, Dilsiz P, Talos DM, Clish CB, Kwiatkowski DJ, Sahin M: Neuronal Tsc1/2 complex controls autophagy through AMPK-dependent regulation of ULK1. Hum Mol Genet 2014;23:3865-3874.

41 Lotz MK, Carames B: Autophagy and cartilage homeostasis mechanisms in joint health, aging and OA. Nat Rev Rheumatol 2011;7:579-587.

-42 Zhang Y, Vasheghani F, Li YH, Blati M, Simeone K, Fahmi H, Lussier B, Roughley P, Lagares D, Pelletier JP, Martel-Pelletier J, Kapoor M: Cartilage-specific deletion of mTOR upregulates autophagy and protects mice from osteoarthritis. Ann Rheum Dis 2015;74:1432-1440.

43 Padmavathi G, Roy NK, Bordoloi D, Arfuso F, Mishra S, Sethi G, Bishayee A, Kunnumakkara AB: Butein in health and disease: A comprehensive review. Phytomedicine 2017;25:118-127.

44 Wang Z, Lee Y, Eun JS, Bae EJ: Inhibition of adipocyte inflammation and macrophage chemotaxis by butein. Eur J Pharmacol 2014;738:40-48.

45 Kojima R, Kawachi M, Ito M: Butein suppresses ICAM-1 expression through the inhibition of IkappaBalpha and c-Jun phosphorylation in TNF-alpha- and PMA-treated HUVECs. Int Immunopharmacol 2015;24:267275.

-46 Semwal RB, Semwal DK, Combrinck S, Viljoen A: Butein: From ancient traditional remedy to modern nutraceutical. Phytochemistry Letters 2015;11:188-201.

47 Pal B, Endisha H, Zhang Y, Kapoor M: mTOR: a potential therapeutic target in osteoarthritis? Drugs R D 2015;15:27-36.

48 Russell RC, Tian Y, Yuan H, Park HW, Chang YY, Kim J, Kim H, Neufeld TP, Dillin A, Guan KL: ULK1 induces autophagy by phosphorylating Beclin-1 and activating VPS34 lipid kinase. Nat Cell Biol 2013;15:741-750.

-49 Petursson F, Husa M, June R, Lotz M, Terkeltaub R, Liu-Bryan R: Linked decreases in liver kinase B1 and AMP-activated protein kinase activity modulate matrix catabolic responses to biomechanical injury in chondrocytes. Arthritis Res Ther 2013;15:1-11.

-50 Zhao X, Petursson F, Viollet B, Lotz M, Terkeltaub R, Liu-Bryan R: Peroxisome proliferator-activated receptor gamma coactivator 1alpha and FoxO3A mediate chondroprotection by AMP-activated protein kinase. Arthritis Rheumatol 2014;66:3073-3082.

51 Zhou S, Lu W, Chen L, Ge Q, Chen D, Xu Z, Shi D, Dai J, Li J, Ju H, Cao Y, Qin J, Chen S, Teng H, Jiang Q: AMPK deficiency in chondrocytes accelerated the progression of instability-induced and ageing-associated osteoarthritis in adult mice. Sci Rep 2017;7:1-14. 